\title{
Soldered Joints of Piezoelectric Products
}

\section{${ }^{\square}$ Olga E. Zheleznikova, ${ }^{2}$ Svetlana A. Mikaeva}

${ }^{1}$ Federal State Budgetary Educational Institution of Higher Education "National Research Mordovian State University named after N. P. Ogaryov", 430005, Republic of Mordovia, Saransk, Bolshevistskaya str., 68 global@ores.su

${ }^{2}$ Federal State Budgetary Educational Institution of Higher Education "MIREA (Moscow Institute of Radio, Electronics and Automatics) - Russian Technological University", 119454, Moscow, Vernadsky Avenue, 78 info@ores.su

\section{Received: 21st August 2020, Accepted: 14th September 2020, Published: 31st October 2020}

\begin{abstract}
The paper considers issues related to the use of capillary luminescent flaw detection for monitoring glass-metal soldered joints of piezoelectric products. Standard methods of leakproofness testing of piezoelectric products are analysed; the framework for the applicability of each of them at the stages of the life cycle of piezoelectric products is established. Based on the results of the studies, a capillary luminescent control method was chosen to check the tightness of glass-metal soldered joints in piezoelectric products. The stages of conducting a capillary luminescent method for monitoring glass-metal soldered joints of piezoelectric products are presented. A method for monitoring the tightness of piezoelectric products using the capillary luminescent method has been developed
\end{abstract}

\section{Keywords}

Piezoelectric Products; Glass-Metal Soldered Joint; Tightness Control; Defects; Capillary Luminescent Control Method; Fiberglass; Input Control

\section{Introduction}

The tightness of piezoelectric products is checked by detecting a gas leakage from the internal cavities of the product when immersed in a liquid with an elevated temperature. This method is suitable for testing sealed piezoelectric products. The liquid used is deionized water heated to the maximum value of the increased operating temperature specified in the technical specifications for the product. Water penetration into a body in the presence of through defects is characterized by the kinematic viscosity of the liquid, which should be no more than $3 \cdot 10^{-6} \mathrm{~m}^{2} \cdot \mathrm{s}^{-1}$ at the test temperature. This method can detect leaks of at least $1 \mathrm{~mm}$. Smaller defects cannot be detected with this method. Such a leak test is effective for products with a large internal volume; they annually lose their relevance in connection with the desire of manufacturers of electrical equipment to reduce their overall dimensions [1].

Another method of leak testing is popular among manufacturers of piezoelectric products; it is used for vacuum products placed in glass-metal cases. This method consists in detecting gas leakage by a mass spectrometer and is used for piezoelectric products having various overall dimensions and internal volumes; it features high reliability and allows detect leaks regardless of the physical and chemical properties inherent in the material of the tested product (Jiashi Yang et al., 2018).

Significant advantages of this method of tightness control are: high sensitivity and the absence of contamination of products during its implementation. The disadvantage is the inability to determine the specific location of the leak. Establishing the exact location of the leak during serial production of products will determine the operation of the technological process, as a result of which the leak has occurred and allow taking measures to eliminate malfunctions in the technological process or introduce additional control operations, if necessary.

The tightness of piezoelectric products is one of the main parameters that guarantee their stable operation within the service life, which averages from 15 to 25 years. Over time, air enters an unpressurized product and the product parameters go beyond the standards established in the technical conditions. The ingress of leaky piezoelectric products into consumer's equipment leads to serious consequences: replacing cells and blocks or rejecting the equipment [3].

\footnotetext{
Materials and Methods

One of the most common reasons for the loss of tightness of piezoelectric products is defects in glass-metal soldered joints, which can be observed under a microscope on the outside of the case under a large magnification. In this regard, an urgent question arose of choosing a method for checking the tightness after the operation of sealing the housings in the area of the glass-metal soldered joint, which makes it possible to determine the relationship between the defects of the joint on the outside of the housing and the product tightness. The appearance of the glass-metal soldered joint without defects is shown in Fig. 1.
} 


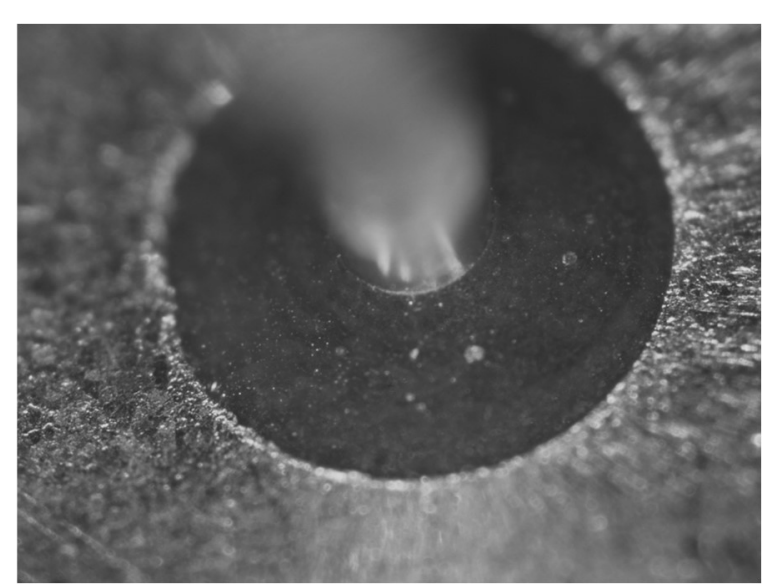

Figure 1: Appearance of Glass-Metal Soldered Joint

Capillary methods were considered to determine the relationship between external defects of a glass-metal soldered joint from the outside of the housing and the product tightness. Unlike standard methods for checking the tightness of piezoelectric products, capillary flaw detection allows us to determine the location on the product surface of invisible or weakly visible defects that cause leaks. The penetrants used in capillary flaw detection have sufficient penetrating power, which allows penetration through the glass-metal soldered joint into the cavity inside the housing. The penetrating ability of penetrants is due to a number of characteristics: penetrant density, optical density of the solution, the ratio of the optical density at a wavelength of $375 \mathrm{~nm}$ to the corresponding optical density at a wavelength of 455 $\mathrm{nm}$. The described feature of the capillary flaw detection will determine the connection of soldered joint defects from the outside of the housing to the tightness of piezoelectric products.

The most common defects of glass-metal soldered joints are cracks, chips, and glass structure violations. The appearance of cracks, chips, and structural defects of the glass-metal soldered joint is shown in Fig. 2.

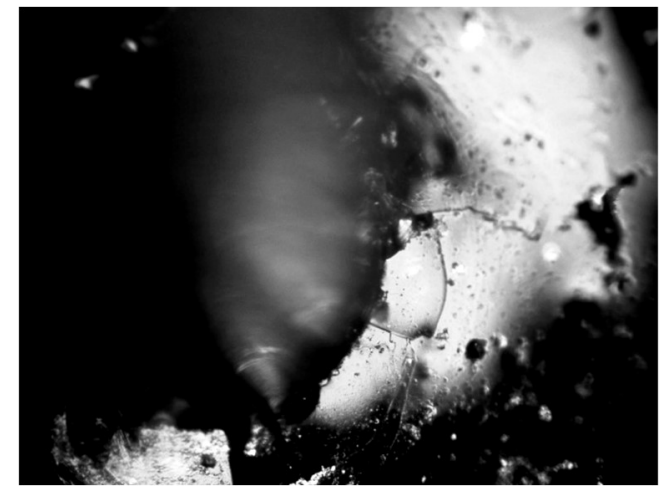

a)

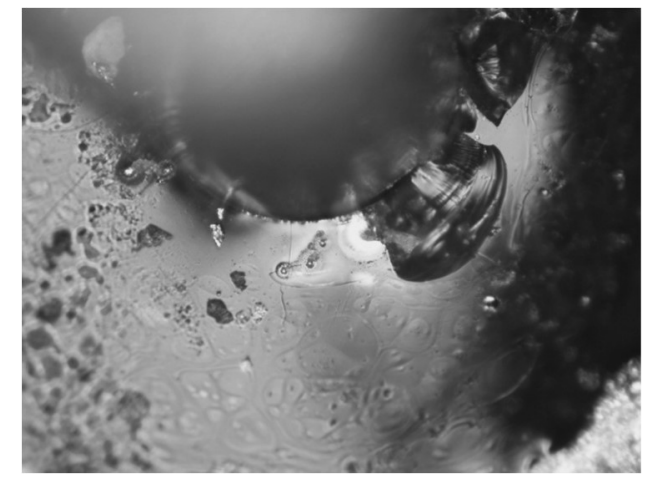

b)

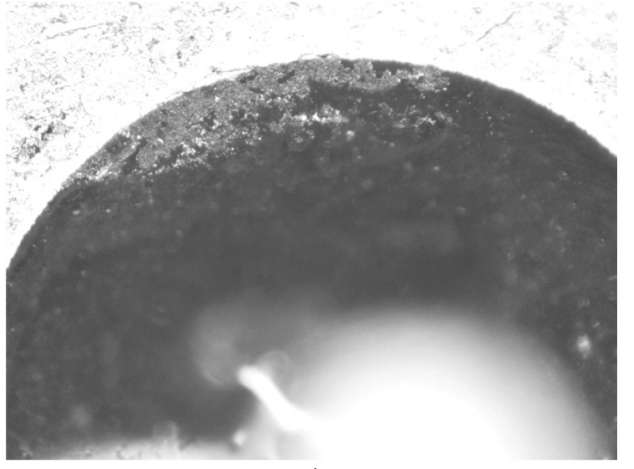

c)

Figure 2: Appearance of Glass-Metal Soldered Joints with Defects: a) Cracks; b) Chips on the Inner Diameter of the Glass Tablet; c) Violation of the Glass Structure at the Outer Diameter of the Glass Tablet 
Violations of the glass structure at the outer diameter of the glass tablet were recorded upon receipt of the housings at the input control. This fact confirms the occurrence of violations in the structure of glass tablets in the manufacturing process of the housings. Cracks and chips of a glass tablet that are visible under a microscope are rare during incoming inspection.

The occurrence of cracks and chips of a glass-metal soldered joint during the production process is due to a violation of the process or the development of internal defects of the glass tablet under the influence of external factors.

During the work, an analysis of defectoscopic materials of the company SPETSAVIA LLC was carried out. The selected LZh-18NV luminescent penetrant for capillary flaw detection has particularly high sensitivity and provides for the detection of cracks on products with depths from 0.01 to $0.03 \mathrm{~mm}$ and more, openings from $0.0005 \mathrm{~mm}$ and more. This penetrant is included in the set of compatible flaw detection materials LUM-330V consisting of penetrant LZh-18NV and cleaning fluid OZh-7A [4].

Penetrant LV-18NV belongs to the 1st sensitivity class [5; 6]. The sensitivity of capillary luminescent flaw detection is determined by the choice of a luminescent material being a substance capable of efficiently converting the energy absorbed by it into visible radiation. Penetrants containing one luminescent material do not reach the maximum possible sensitivity; therefore, they use two or more luminescent materials to increase the sensitivity of penetrants. The composition of the penetrant LZh-18NV includes two luminescent materials interacting according to the principle of "cascade luminescence". This principle consists in the intermolecular energy transfer of electronic excitation.

\section{Result and Discussion}

Thus, it is advisable to carry out capillary luminescent control of a glass-metal soldered joint in several stages:

- The first stage consists in preparing the bases of the housings of the piezoelectric products for control; this includes chemical cleaning the surface of the bases of the housings from various contaminants and their subsequent drying. Cleaning is carried out by treatment of the controlled bases of the housings with an aqueous solution of acetone; and their drying is carried out in an air stream at an elevated temperature $\left(90^{\circ} \mathrm{C}\right.$ for 20 minutes);

- The second stage is the processing of glass-metal soldered joints of the tested products with the penetrant. 2 methods of applying the penetrant are selected for the process. The first method is a capillary one; it consists in immersing the controlled housings into the penetrant. It was established that the average time spent by the housings in the penetrant is several days; it ensures filling of the defective area with it. The disadvantage of this method of applying penetrant is the long time spent on penetrating it into the capillaries. The second application method is ultrasonic, where the time for which the penetrant penetrates the defects of a glass-metal soldered joint is significantly reduced. In the process of work, an increase in the width and depth of cracks of the glass tablet under the ultrasonic influence was found. If the glass-metal soldered joint is free of defects, then ultrasound does not provoke their occurrence;

- At the third stage, the selected penetrant is removed by washing under a stream of water, the temperature range of which is from 18 to $38^{\circ} \mathrm{C}$. Controlled products after washing are rubbed with calico and dried in a stream of air; - The next stage is the cleaning of controlled products from penetrant residues with liquid OZh-7A;

- At the final stage of control, the parameters of the products are measured. We can clearly see the through defects in the glass-metal soldered joint after opening the products. The control is carried out under a Shuttlepix P-400R digital microscope in ultraviolet light $[7 ; 8 ; 9 ; 10]$.

\section{Conclusion}

It has been established that important advantages of the capillary luminescent control method are: detection of through and surface defects, determination of their location and extent, the ability to control products of various shapes and sizes made of various materials (metals, alloys, glass, etc.), high sensitivity, and low cost of purchased reagents.

This method is applicable for tightness control in areas of defects of glass-metal soldered joints and weld seams in the housings of piezoelectric products. The use of the capillary luminescent control method for checking the tightness of glass-metal housings was first implemented by the company LIT-FONON JSC and is recommended for testing piezoelectric products after the sealing operation.

As part of the work, a method for monitoring the tightness of piezoelectric products was developed, including the use of the capillary luminescent method.

\section{References}

[1] Ruoran Cheng, Chunli Zhang, Weiziu Chen, Jiashi Yang, 2019. The temperature effect on mobile charges when tensioning composite fibres of piezoelectric dielectrics and non-piezoelectric semiconductors. International Journal of Applied Mechanics, 11: 9.

[2] Jiashi Yang et al., 2018. The energy conversion efficiency of a piezoelectric thermoelectric material. 2018. Journal of Electronic Materials, 47: 8. 
[3] Ruoran Cheng, Chunli Zhang, Weiziu Chen, Jiashi Yang, 2018. Piezotron effects in tension of a composite fibre made of piezoelectric dielectrics and non-piezoelectric semiconductors. Journal of Applied Physics, 124: 6 - 9.

[4] Mikaeva A.S., 2019. Modern electronic systems and devices. RUSINES: M., p.186.

[5] Babenko V.P., Bitykov V.K., Kuznetsov V.V., 2018. Simulation of static and dynamic losses in mosfet keys. Russion Technological Journal, 6(1): 20 - 39.

[6] Ashryatov A.A., Fedorenko A.S, 2012. On further increasing the luminous efficacy of low pressure fluorescent lamps. Light \& Engineering, 20 (2): 93 - 98.

[7] Jian Li, Chun-li Zhang, Rong-hao Bao, Wei-qiu Chen, 2019. Research laboratory on the mechanics of smart materials and structures, Zhejiang University. Journal of Zhejiang University-SCIENCE A, 20(4): 305-310.

[8] Wanli Yang, Shuaiqi Fan, Yuxing Liang, Yuantai Hu, 2019. Prestress-loading effect on the current-voltage characteristics of a piezoelectric $\mathrm{p}-\mathrm{n}$ soldered joint together with the corresponding mechanical tuning laws. Beilstein Journal of Nanotechnology, 10:1833-1843.

[9] Guolin Wang, Jinxi Liu, Xianglin Liu, Wenjie Feng, Jiashi Yang, 2018. Extensional vibration characteristics and screening of polarization charges in a $\mathrm{ZnO}$ piezoelectric semiconductor nanofiber. Journal of Applied Physics, 124: $9-11$.

[10] Ruoran Cheng, Chunli Zhang, Weiqiu Chen, Jiashi Yang, 2020. Temperature Effects on PN Soldered joints in Piezoelectric Semiconductor Fibers with Thermoelastic and Pyroelectric Couplings. Journal of Electronic Materials, 43:10-13. 